\title{
Lane-Level Vehicle Trajectory Reckoning for Cooperative Vehicle-Infrastructure System
}

\author{
Yinsong Wang, Xiaoguang Yang, Luoyi Huang, and Jiawen Wang
}

Key Laboratory of Road and Traffic Engineering of the Ministry of Education, Tongji University, 4800 Cao'an Road, Shanghai 201804, China

Correspondence should be addressed to Yinsong Wang, pinysong@163.com

Received 11 September 2012; Revised 26 November 2012; Accepted 27 November 2012

Academic Editor: Wuhong Wang

Copyright (C) 2012 Yinsong Wang et al. This is an open access article distributed under the Creative Commons Attribution License, which permits unrestricted use, distribution, and reproduction in any medium, provided the original work is properly cited.

This paper presents a lane-level positioning method by trajectory reckoning without Global Positioning System (GPS) equipment in the environment of Cooperative Vehicle-Infrastructure System (CVIS). Firstly, the accuracy requirements of vehicle position in CVIS applications and the applicability of GPS positioning methods were analyzed. Then, a trajectory reckoning method based on speed and steering data from vehicle's Control Area Network (CAN) and roadside calibration facilities was proposed, which consists of three critical models, including real-time estimation of steering angle and vehicle direction, vehicle movement reckoning, and wireless calibration. Finally, the proposed method was validated through simulation and field tests under a variety of traffic conditions. Results show that the accuracy of the reckoned vehicle position can reach the lane level and match the requirements of common CVIS applications.

\section{Introduction}

The recently developed Cooperative Vehicle-Infrastructure System (CVIS) enables vehicle with the capability to communicate with other vehicles and infrastructure and to ensure safety and travel efficiency by driving assistance [1]. The capability of providing accurate position estimation is essential for successful and widespread deployment of CVIS applications such as optimal speed advisory and lane change assistant.

The Global Positioning System (GPS) is one of the most convenient and accurate methods for determining vehicle position in a global coordinate system [2, 3]. Low-cost GPS receivers can provide positioning service with an accuracy of approximately 10-20 meters, and the Differential GPS (DGPS) receivers have improved the positioning accuracy to approximately 2-3 meters, but require high construction cost for both onboard receiver and base station. To realize lane-level positioning, vehicle movement dynamics was integrated 
into the position estimate by an Extended Kalman filter utilizing only low-cost GPS equipment, and the GPS errors were estimated from an event-driven vehicle turn and lanealignment optimization model based on an accurate GPS description of the roadway [4]. In addition, a vehicle-lane-determining system was described in [5], which consists of an onboard DGPS receiver connecting with a wireless communications channel, a unique lanelevel digital roadway database, a developed lane-match algorithm, and a real-time vehicle location display. Deduced Reckoning (commonly referred to as "Dead" Reckoning or DR) sensors consisting of an odometer and a gyroscope are routinely used to bridge any gaps in GPS positioning [6]. This information is then used with spatial road network data to determine the spatial reference of vehicle location via a process known as map matching [7]. Toledo-Moreo presented a solution that integrated a Global Navigation Satellite System (GNSS) receiver, an odometer, and a gyroscope, along with the road information stored in enhanced digital maps to analyze the reckoning trajectory [8].

On the other hand, most of the CVIS applications do not need the vehicle position within a global coordinate system. Positioning methods in relative coordinate systems such as Radio Frequency Identification (RFID) were developed recently. Accurate indoor position estimation utilizing passive RFID has been realized [9, 10]. In road traffic, Lee proposed a RFID-assisted localization system, which employed the DGPS concept to improve GPS accuracy [11]. Scheme of active RFID positioning of vehicles was developed in [12], the RFID tags were installed along lanes on road to form RFID matrix and the vehicle equipped with RFID readers locate itself by retrieving position information from tags around it.

This paper explores lane-level positioning by vehicle trajectory reckoning utilizing speed and steering data from vehicle's Control Area Network (CAN). In addition, since the method was deployed in CVIS environment, the roadside calibration facility was designed by utilizing Detected Short Range Communication (DSRC) technology to improve the trajectory reckoning.

\section{Accuracy Requirements for Positioning}

\subsection{The CVIS Environment}

In a previous publication [13], a proof of concept for a prototype of Cooperative VehicleInfrastructure System (TJ-CVIS) developed at the University of Tongji was presented, which provides a vehicle with the capability to communicate with other vehicles and infrastructure. Conceptually, by using sensor technologies and standardized communication protocol, the vehicle probe data such as position, velocity, and preplanned routes can be automatically collected with high frequency and accuracy in CVIS. Therefore, a series of advanced traffic control applications and advanced driving assist applications for safety and efficiency can be deployed relying on the high sensitivity and information interactive abilities of CVIS. Table 1 lists the most common CVIS applications and their accuracy requirements for positioning [14].

\subsection{GPS Position Errors}

Low-cost GPS equipment has been widely used in the navigation applications. However, as the analysis shown in Section 2.1, the accuracy requirements of navigation and CVIS 
Table 1: Typical CVIS applications and positioning accuracy requirements.

\begin{tabular}{lcc}
\hline Application & Location/direction & Accuracy requirements \\
\hline$\checkmark$ Route navigation & Vehicle heading & Road section level \\
$\checkmark$ Optimal speed advisory & Vehicle heading & Lane level \\
$\checkmark$ Automatic cruise & Vehicle heading & Lane level \\
$\checkmark$ Lane change assist & Lane heading & Lane level \\
$\checkmark$ Overtaking assist & Lane heading & Lane level \\
$\checkmark$ Collision avoidance & At intersection & Lane level \\
$\checkmark$ Active traffic control & At intersection & Lane level \\
\hline
\end{tabular}

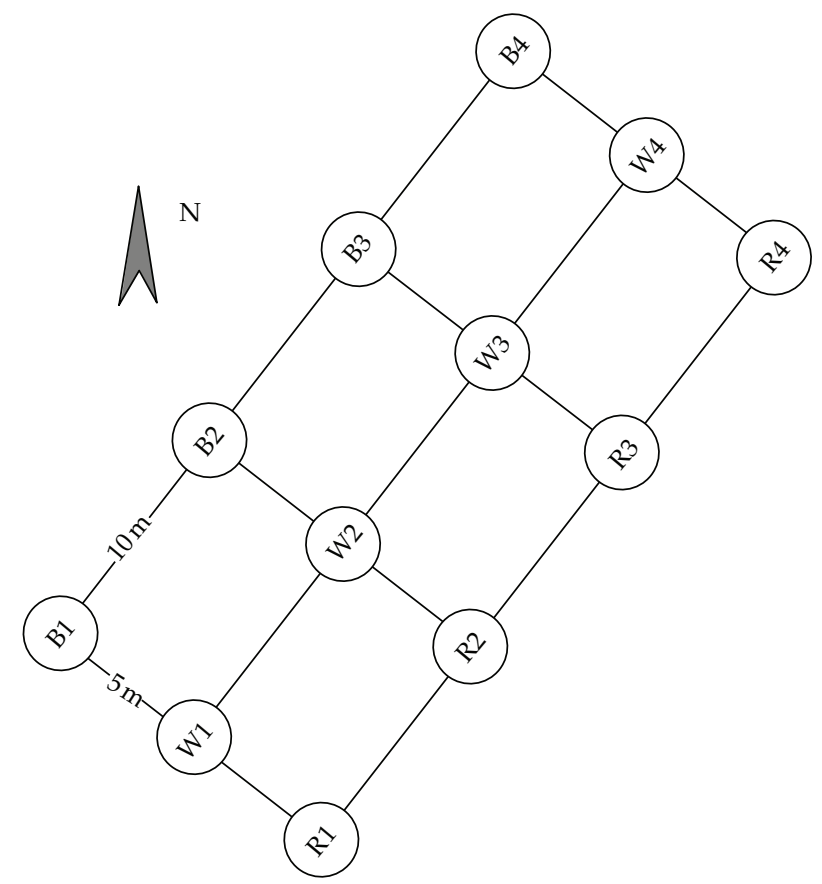

Figure 1: Actual shape of test grid.

applications are markedly different. In order to test the feasibility of low-cost GPS equipment in CVIS, a common GPS receiver was placed at a set of stationary positions which form a grid shown in Figure 1. The positioning result is shown in Figure 2.

In Figure 2, the test result is totally out of shape compared to the actual shape of test sites. The distance between any two points in a line or row was calculated by the GPS data. The result shown in Table 2 indicates that in the situation of stationary positioning, the GPS errors arrive at $2.79 \mathrm{~m}$ on average, which is inapplicable for the CVIS applications.

In order to test the dynamic positioning accuracy of GPS, we conducted a test on an urban road, the starting and completing time stamps of lane changing were manually recorded. Figure 3 shows an example of dynamic positioning test in the situation of lane change. Results show that the performance of dynamic positioning is better than static 


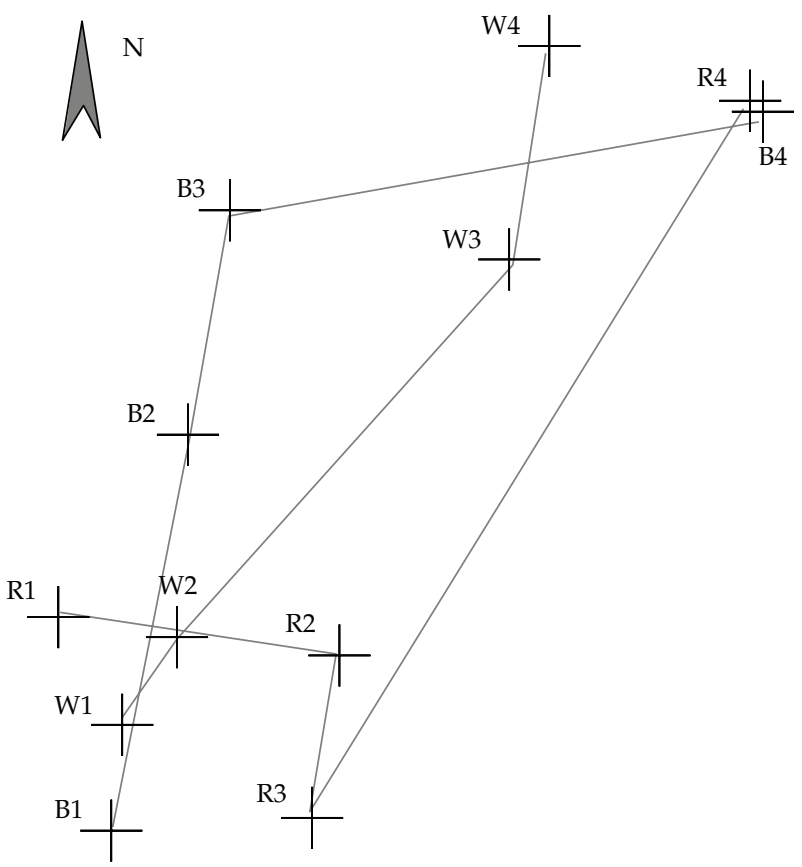

Figure 2: Stationary test result.

Table 2: Stationary positioning accuracy of GPS.

\begin{tabular}{lccccc}
\hline From & To & Calculated distance $(\mathrm{m})$ & Actual distance $(\mathrm{m})$ & Error $(\mathrm{m})$ & Proportional error \\
\hline B1 & B2 & 27.12 & 10.00 & -17.12 & $171.16 \%$ \\
B2 & B3 & 7.47 & 10.00 & 2.53 & $25.25 \%$ \\
B3 & B4 & 6.57 & 10.00 & 3.43 & $34.35 \%$ \\
B4 & B1 & 23.14 & 30.00 & 6.86 & $22.86 \%$ \\
W1 & W3 & 21.85 & 20.00 & -1.85 & $9.25 \%$ \\
W1 & W4 & 30.22 & 30.00 & -0.22 & $0.74 \%$ \\
W3 & W2 & 16.26 & 10.00 & -6.26 & $62.57 \%$ \\
W4 & W1 & 30.22 & 30.00 & -0.22 & $0.74 \%$ \\
R1 & R2 & 10.88 & 10.00 & -0.88 & $8.83 \%$ \\
R2 & R3 & 13.48 & 10.00 & -3.48 & $34.84 \%$ \\
R3 & R4 & 26.99 & 10.00 & -16.99 & $169.90 \%$ \\
R4 & R1 & 31.28 & 30.00 & -1.28 & $4.27 \%$ \\
R4 & R3 & 10.90 & 10.00 & -0.90 & $9.04 \%$ \\
\hline
\end{tabular}

positioning [13]. Although map-matching can improve the positioning accuracy if good spatial road network data are available [15], a poor-quality road map could lead to a large error in map-matched solutions. Hence the lane changing event still cannot be easily recognized in the normal traffic environment. 


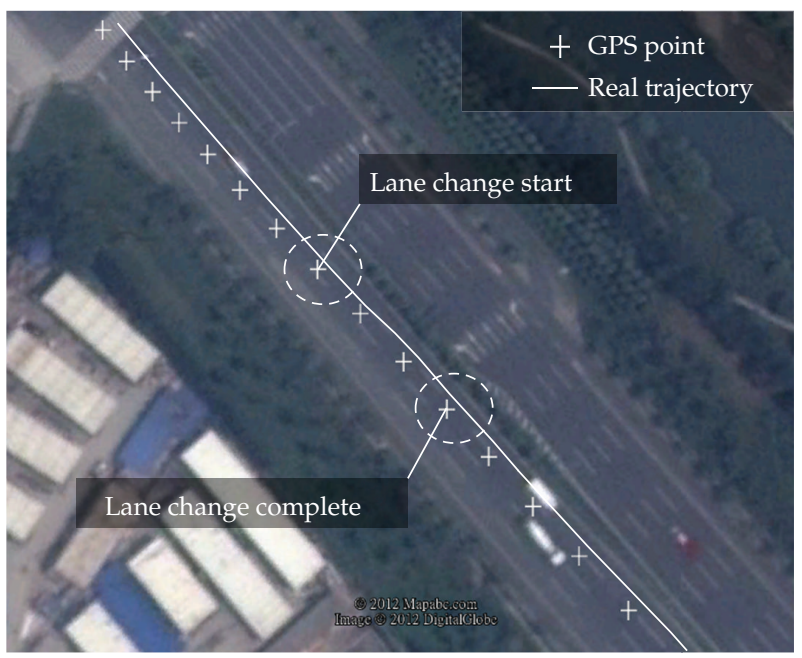

Figure 3: Dynamic positioning test of GPS.

\section{Vehicle Trajectory Reckoning}

\subsection{Input Parameters}

The idea of lane-level positioning in this paper is reckoning the vehicle trajectory based on vehicle's driving-related data, then determining the position of vehicles. Figure 4(a) shows the actual vehicle trajectory of a left lane change event, Figures 4(b) and 4(c) show the variations of steering wheel angle and the front wheels' steering angle corresponding to the lane change event described in Figure 4(a). According to the data from CAN, the speed, steering wheel angle, and the wheels' steering angle can be recorded with a high frequency of $100 \mathrm{~Hz}$, which enables the possibility to reckon the trajectory of a moving vehicle. Therefore, the vehicle position can be determined by the precise trajectory.

Since vehicle's body shape bears on the reckoning input, it is necessary to define the reference point of the vehicle. In this study, we developed the trajectory reckoning algorithm based on the four-wheel vehicle with a wheel track of $B$ and a wheel base of $L$ shown in Figure 5. For the four-wheel vehicle, each front wheel should perform only rolling movement around the rotation center $O$ when steering. As an example of right steering shown in Figure 5, steering angle of the inner wheel $(\beta)$ should be greater than the outer wheel $(\alpha)$. The relationship between $\beta$ and $\alpha$ is described as the following:

$$
\cot \alpha=\cot \beta+\frac{B}{L}
$$

To simplify the algorithm, we use the point of rotation axis of left-front wheel as the reference point shown as $P$ in Figure 5. Finally, the input parameters can be specified as the vehicle body shape parameters, left-front wheel's speed $(v)$, and its steering angle $(\alpha)$. For the vehicles which can only output the steering wheel angle $(\gamma), \alpha$ can be calculated by $\alpha=f(\gamma)$, which is specified by the vehicle type. Figure 6 shows an example of $f(\gamma)$ tested by a common four-wheel vehicle. 


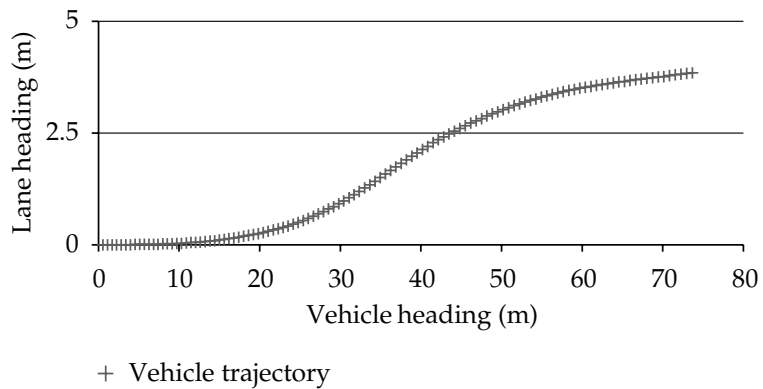

(a) Vehicle trajectory

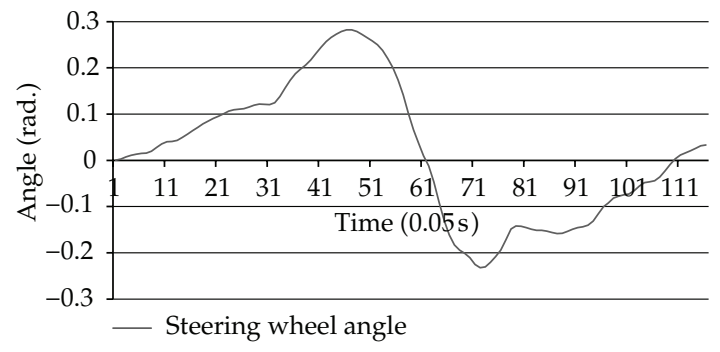

(b) Steering wheel angle

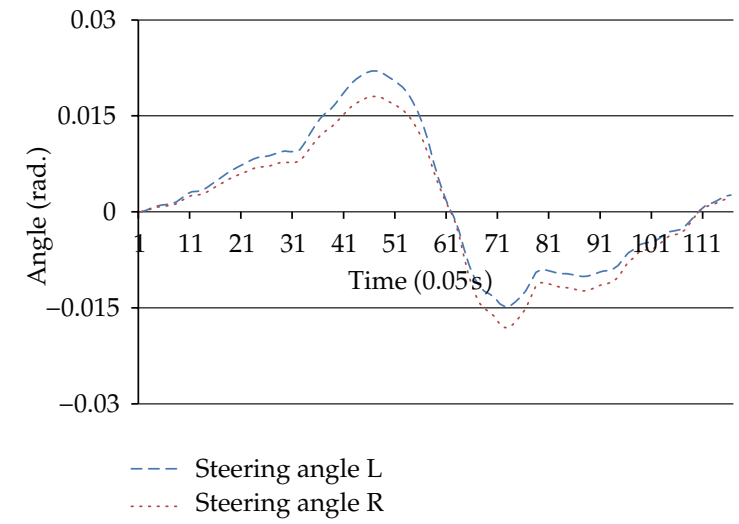

(c) Steering angle

Figure 4: Variations of parameters in lane change event.

\subsection{Trajectory Reckoning}

Since GPS is not utilized in our test, which means global coordinate system is not available for the trajectory reckoning method, we choose the dynamic-update coordinate system shown in Figure 7. The calibration method will be introduced in Section 3.3.

At any time, the rotation radius $(R$, in meters $-\mathrm{m})$ of the reference point can be calculated as the following:

$$
R= \begin{cases}\frac{L}{\sin (|\alpha|)}, & |\alpha|>0 \\ \infty, & |\alpha|=0 .\end{cases}
$$




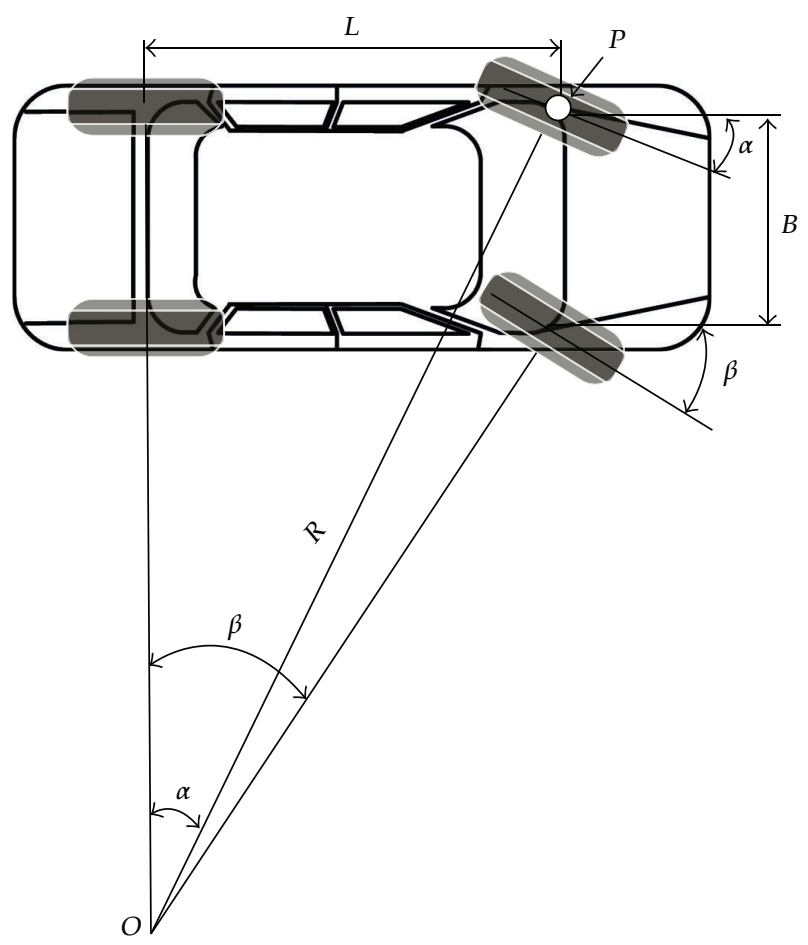

Figure 5: Optimal relationship of steering angle.

Angular speed ( $\mu$, in radian/second-rad/s) of reference point as the following:

$$
\mu= \begin{cases}\frac{v}{R}, & \alpha>0 \\ 0, & \alpha=0 \\ -\frac{v}{R}, & \alpha<0 .\end{cases}
$$

In (3.3), $v$ is the linear speed of the reference point (in meters/second- $m / s$ ). When the trajectory is being reckoning, $v$ and $\alpha$ are sampled with high frequency. Thus, the rotation angle ( $\omega$, in radian-rad) of the vehicle body can be calculated as the following:

$$
\omega=\sum \mu_{i} \times t
$$

In (3.4), $\mu_{i}$ denotes the angular speed calculated by (3.3) at time sequencei, and $t$ is the sampling interval.

The movements refer to the calibration point as:

$$
\begin{aligned}
& \Delta x_{i}=v_{i} \times \cos \omega \times t \\
& \Delta y_{i}=v_{i} \times \sin \omega \times t .
\end{aligned}
$$




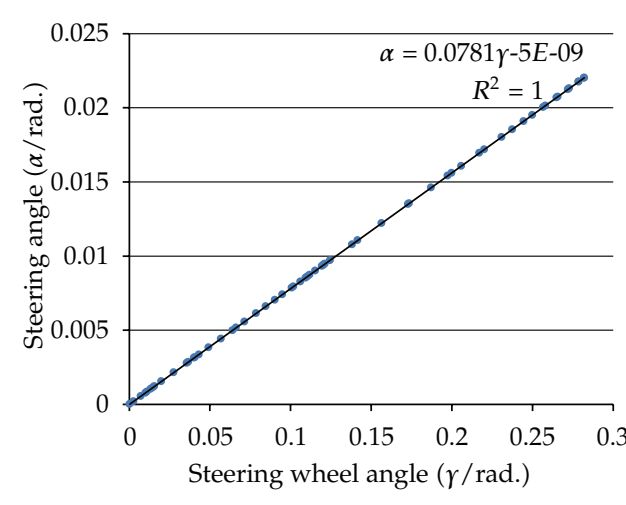

- Left steering

(a) Left steering $(\gamma>0)$

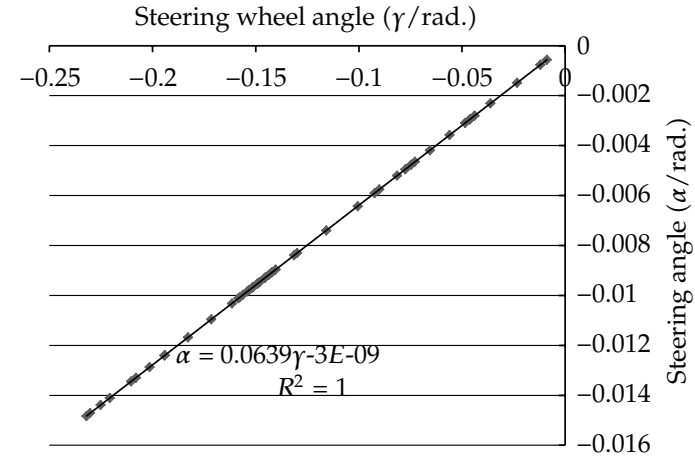

- Right steering

(b) Right steering $(\gamma<0)$

Figure 6: Example of $f(\gamma)$.

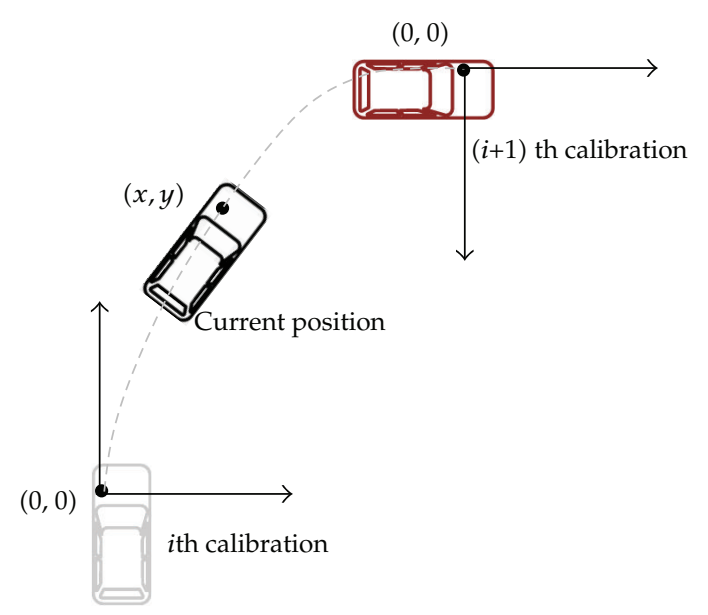

Figure 7: Dynamic update coordinate system.

In (3.5), $v_{i}$ denotes the speed output at time sequence $i$, and the vehicle position at time sequence $i$ can be reckoned as follows:

$$
\begin{aligned}
& x_{i}=\sum_{j=0}^{i} \Delta x_{j}, \\
& y_{i}=\sum_{j=0}^{i} \Delta y_{j} .
\end{aligned}
$$




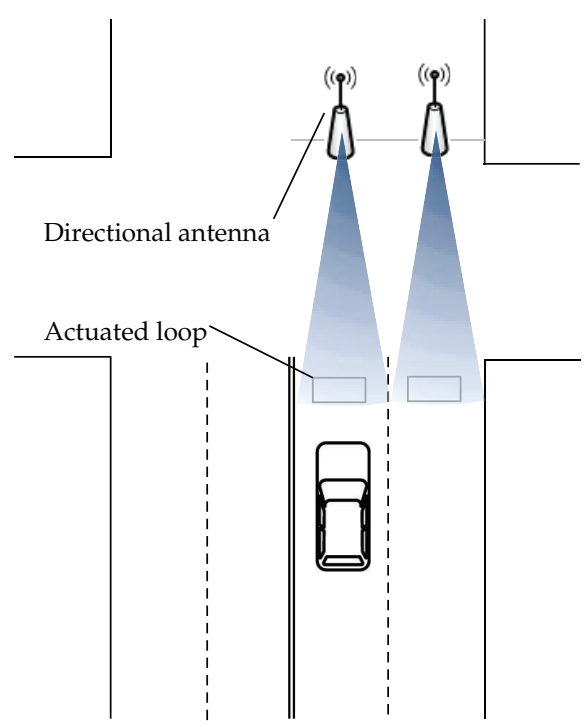

(a) Calibration facilities (top view)

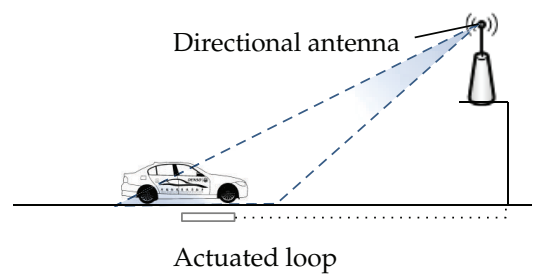

(b) Calibration facilities (side view)

Figure 8: Calibration facilities.

\subsection{Calibration Method}

For the data output from CAN, the random errors cannot be avoided, and the cumulative errors in reckoning results increased rapidly when the distances get longer. It is necessary to calibrate the trajectory at a certain distance. Once the trajectory is calibrated, the dynamicupdated coordinate system referred above is updated.

The CVIS environment provides a vehicle with the capability to communicate with roadside infrastructures, which can be adopted to calibrate the vehicle trajectory. Figure 8 indicates the design of calibration facilities. The actuated loop, which has been already installed in most signalized intersections, is utilized in this method to detect the passing of vehicle. The directional antenna is set to send the calibration information to the vehicle by DSRC when vehicle is passing through the loop [16]. The road geometry data including road curvature, intersection geometry and lane drop geometry are stored in the onboard unit. In the prototype of TJ-CVIS [13], the content of calibration information was specified as the following:

$\checkmark$ actuate time;

$\checkmark$ road section ID;

$\checkmark$ loop ID;

$\checkmark$ lane ID of the loop;

$\checkmark$ distance to the downstream calibration points.

With the calibration information, vehicle's position can be determined by comparing the reckoned trajectory with road geometry data. We assume that the vehicle is not 


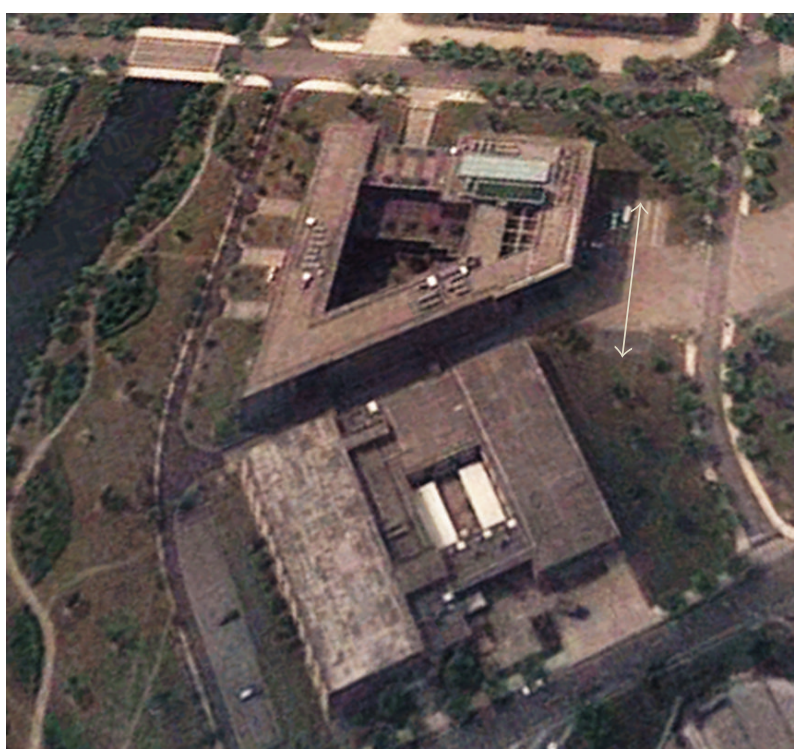

Figure 9: Field test Scenario 1.

steering when passing a loop at intersections. The calibration procedure is described as the following.

Step 1. Calibration message received by the vehicle.

Step 2. Update coordinate system referring to loop ID, lane ID and road section ID,

Step 3. $x_{i}=v_{i} \times t_{\text {latency, }} y_{i}=0, \omega=0$. ( $t_{\text {latency }}$ denotes the constant value of wireless communication latency, $t_{\text {latency }}=0.01 \mathrm{~s}$ in the prototype of TJ-CVIS [13]).

Step 4. Keep trajectory reckoning until next calibration.

\section{Field and Driving Simulator Trials}

\subsection{Field Test}

In order to validate the trajectory reckoning method, we conducted a field test at the campus of Tongji University. A computer program was developed on Windows platform to collect output data from CAN and reckon the trajectory. A common four-wheel vehicle was utilized as the experiment vehicle. Since the data protocol of CAN has not been disclosured by the manufacturer, we made use of the On-Board Diagnostics (OBD) protocol to read the vehicle speed. Hence only the algorithm for vehicle heading movement was validated in field test.

The first scenario of field test was deployed at a flattened square at the campus shown in Figure 9. The experiment vehicle was driven to pass through a pre-measured road segment with $37.5 \mathrm{~m}$ long. Another scenario of field test was deployed at a $276.6 \mathrm{~m}$-long road shown in Figure 10. When experiment vehicle entering the test segment, the on-board unit started to reckon the trajectory. And when experiment vehicle leaving test segment, the distances of 


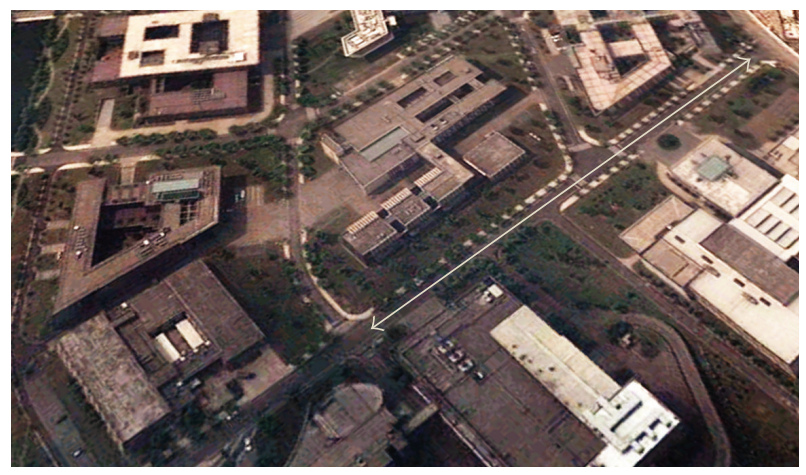

Figure 10: Field test Scenario 2.

Table 3: Result of field test Scenario 1 (5 trials).

\begin{tabular}{lcccc}
\hline ID & Actual distance $(\mathrm{m})$ & Reckoned distance $(\mathrm{m})$ & Error $(\mathrm{m})$ & PE \\
\hline 1 & 37.50 & 37.29 & -0.21 & $0.56 \%$ \\
2 & 37.50 & 37.56 & 0.06 & $0.16 \%$ \\
3 & 37.50 & 37.83 & 0.33 & $0.88 \%$ \\
4 & 37.50 & 37.33 & -0.17 & $0.45 \%$ \\
5 & 37.50 & 37.33 & -0.17 & $0.45 \%$ \\
\hline
\end{tabular}

Table 4: Result of field test Scenario 2 (10 trails).

\begin{tabular}{lccr}
\hline Actual distance $(\mathrm{m})$ & Average reckoned distance $(\mathrm{m})$ & ME $(\mathrm{m})$ & MPE \\
\hline 276.60 & 277.54 & -0.94 & $0.34 \%$ \\
\hline
\end{tabular}

trajectory between the vehicle's current position and the segment start line were recorded. The sampling frequency is $5 \mathrm{~Hz}$ in both scenarios. Test results are shown in Tables 3 and 4.

Results from field test indicate that the trajectory reckoning method can reach the submeter accuracy both in the two scenarios. It can also certify that the data from CAN are applicable for the reckoning method. Since the speed accuracy output by OBD protocol is limited to $\pm 1 \mathrm{~km} / \mathrm{h}(0.28 \mathrm{~m} / \mathrm{s})$, the reckoning accuracy will be improved if we use the standard CAN protocol, which has a nominal output speed accuracy of $\pm 0.01 \mathrm{~km} / \mathrm{h}$ $(0.003 \mathrm{~m} / \mathrm{s})$.

\subsection{Driving Simulator Trials}

Another validation was taken on driving simulator due to the lack of CAN communication protocol of real vehicles. The driving simulator involved in validation was constructed by Tongji University, including a movement system with eight degrees of freedom shown in Figure 11. A real four-wheel vehicle with real steering system was installed in the movement system to simulate the situation of steering, braking, and acceleration. By using the driving simulator, the steering angle and speed can be sampled with high accuracy. Moreover, the actual trajectory can also be recorded for every sampling interval shown in Figure 12, which 


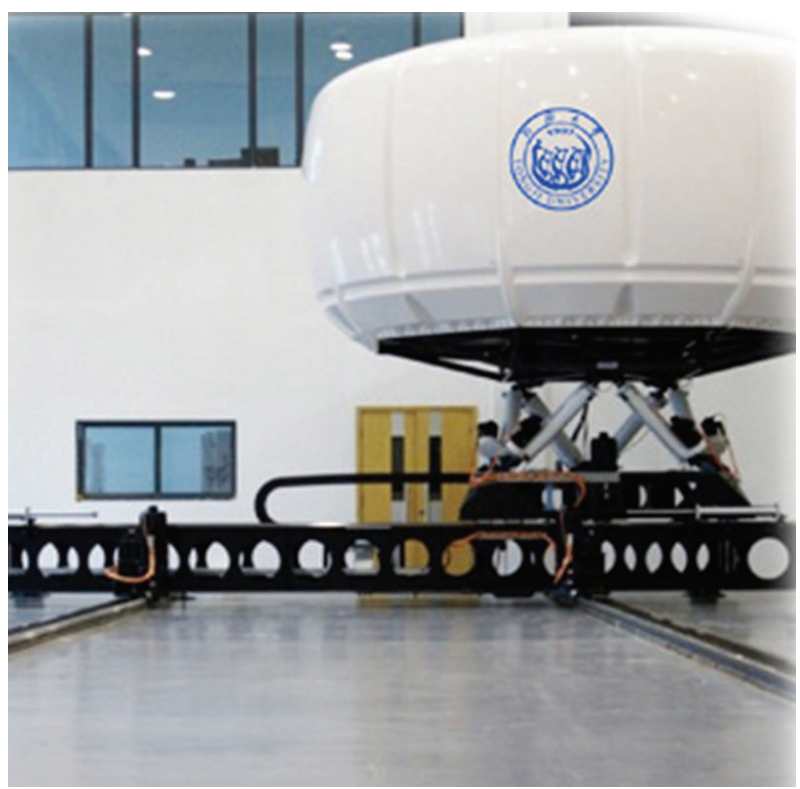

Figure 11: Driving simulator in Tongji University.

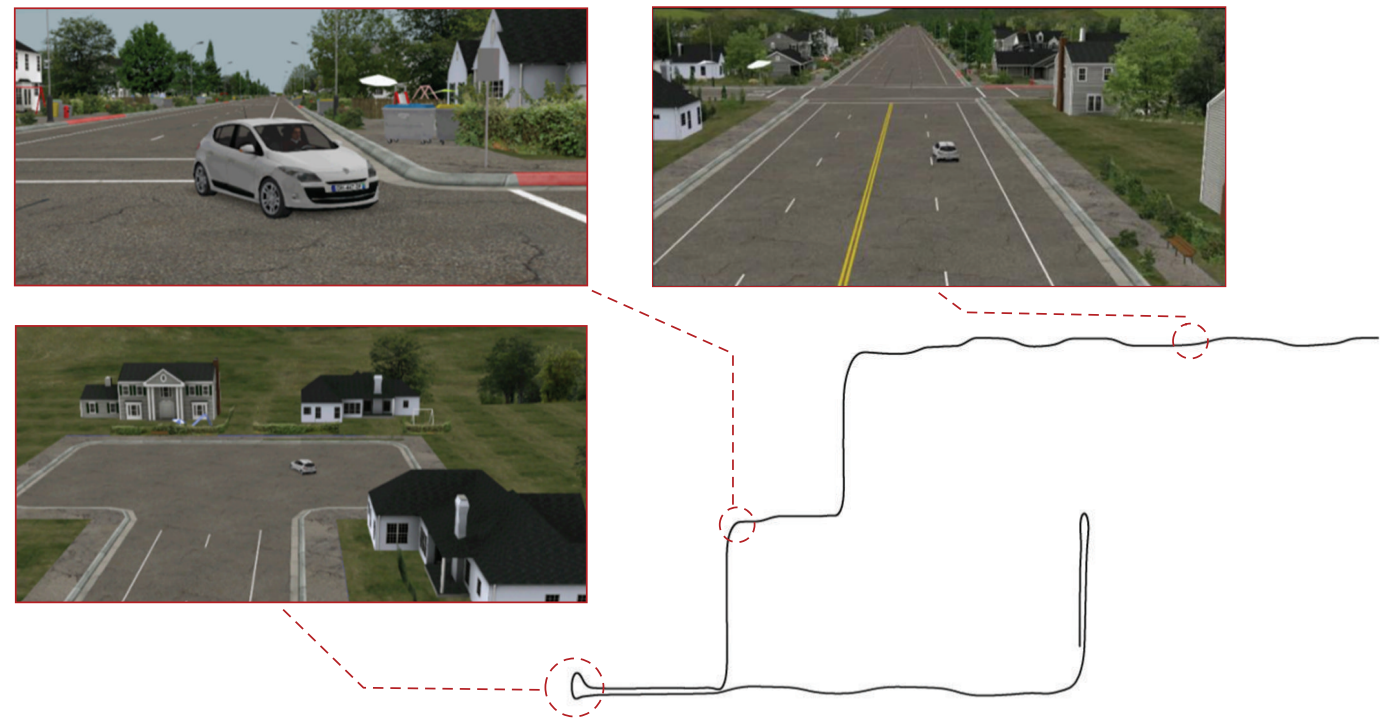

Figure 12: Actual trajectory and driving scenarios in driving simulator.

is hardly to record in field test and can make it easier to analyze the trajectory reckoning errors.

In the driving simulator trials, the driver was asked to change lane and turn at different speeds. The speed and steering angle of front-left wheel were recorded every $0.05 \mathrm{~s}$. According to the manufactory, the wheel base $(L)$ is $2.686 \mathrm{~m}$. In addition, virtual calibration points were set at outbound lanes of each intersection. Examples of lane change trajectory and turning trajectory reckoning are shown in Figures 13 and 14. 


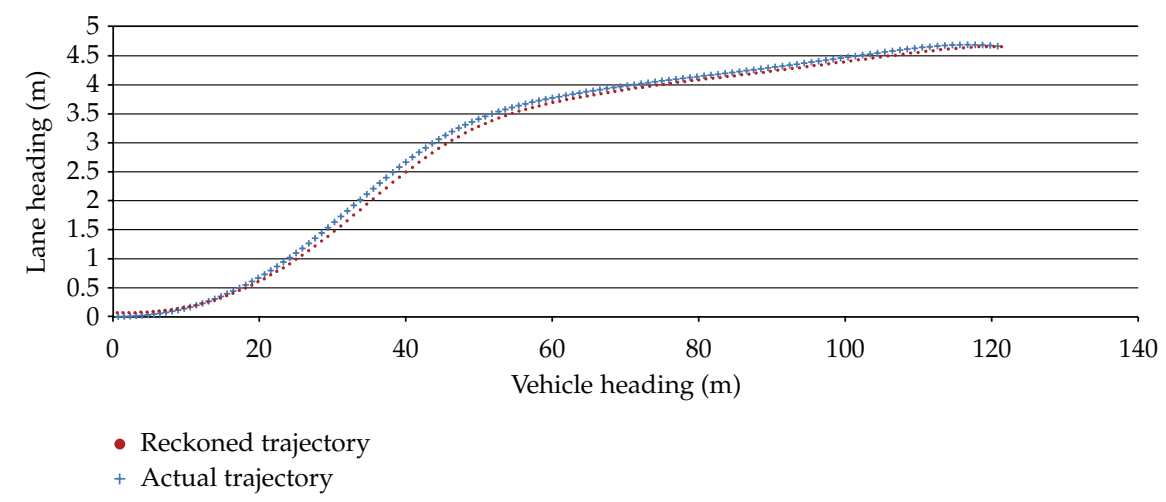

(a) Trajectory reckoning result

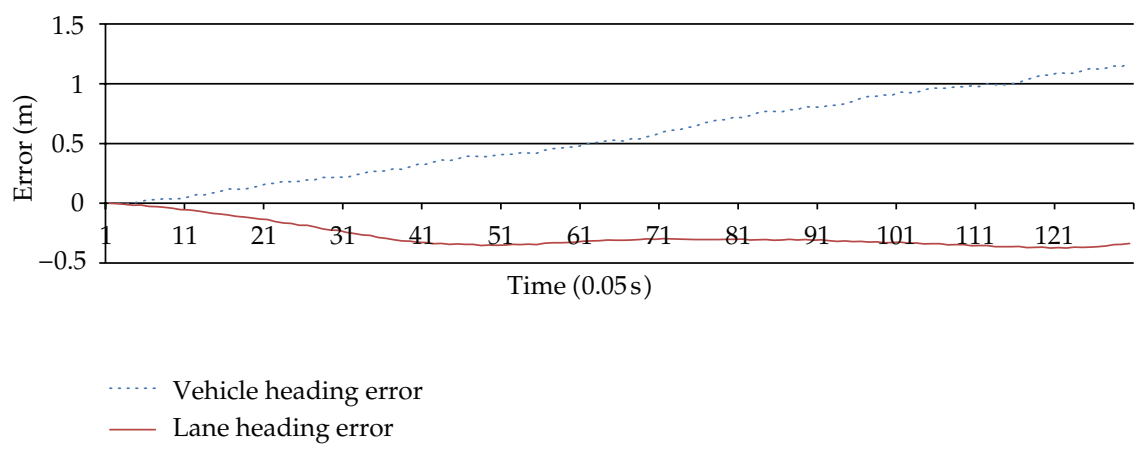

(b) Reckoning error

Figure 13: Example of trajectory reckoning result of lane change.

The two examples indicate that the reckoning results are very close to the actual trajectories. If the geometry of the road and lane drop are known, the position of vehicle (i.e., distance to the next stop line and in which lane the vehicle is driving) can be determined by the reckoned trajectory.

Table 5 shows the statistical result of driving simulator trials, indicating that the proposed trajectory reckoning method can reach the accuracy of submeter both in vehicle heading and lane heading directions ( $\mathrm{U}$ turn is not included in the statistic).

\section{Conclusions and Future Work}

This paper introduced a lane-level positioning method by trajectory reckoning without GPS equipment in the environment of CVIS. Vehicle driving data from CAN was adopted to reckon the vehicle movements, and then the position was determined by the reckoned trajectory object to the road geometry. In addition, a calibration method was designed to eliminate the cumulate errors during long-time reckoning. Results from field tests and driving simulator indicate that the proposed method can reach the positioning accuracy of submeter, which meets the requirements of most CVIS applications. Hence, the proposed method can be further deployed in the real traffic. 


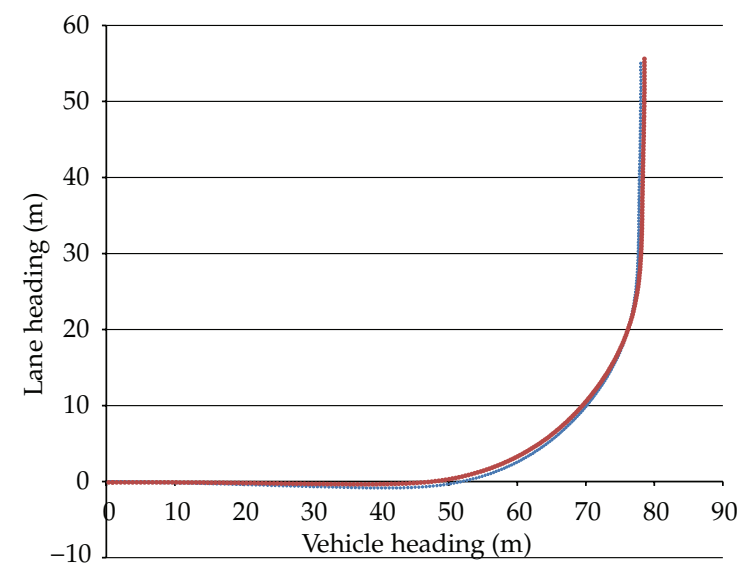

- Reckoned trajectory

+ Actual trajectory

(a) Trajectory reckoning result

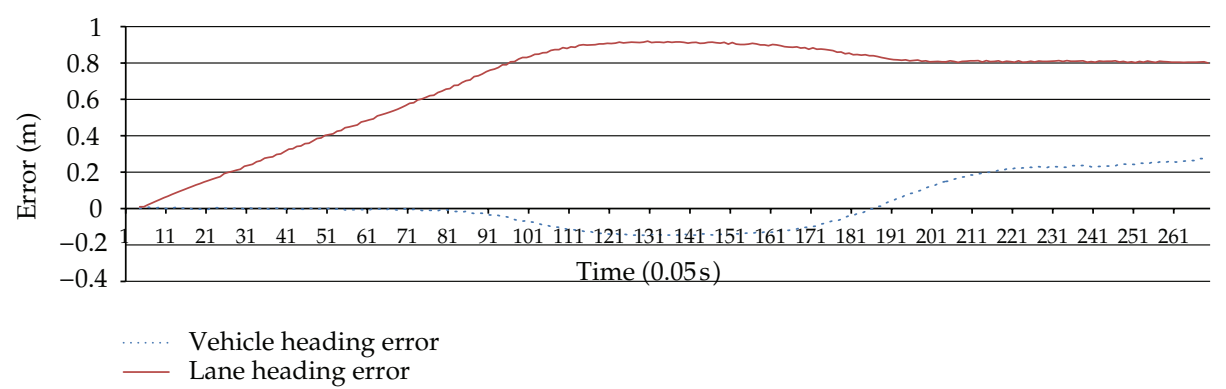

(b) Reckoning error

Figure 14: Example of trajectory reckoning result of turning.

Table 5: Trajectory reckoning results.

\begin{tabular}{lccc}
\hline Event & Number of trials & MAE of $x$ (vehicle heading, $\mathrm{m}$ ) & MAE of $y$ (lane heading, $\mathrm{m}$ ) \\
\hline Lane change & 13 & 0.56 & 0.27 \\
Turning & 5 & 0.03 & 0.58 \\
\hline
\end{tabular}

Although the method outperforms GPS, based on the field test and driving simulator trials, it has certain limitations. For instance, the system errors increase as the increase of length of trajectory takes place, and the performance of calibration facility drops when too many vehicles approach, which deserve further discussion.

\section{Acknowledgments}

This paper was made possible through funding from the Ministry of Science and Technology of China under Grant 2011AA110404 and the National Natural Science Foundation of China 
under Grant 60974093. And the authors wish to acknowledge the vast and helpful assistance from Cooperative Vehicle-Infrastructure System Lab and the Driver Behavior and Traffic Safety Simulation Platform (Tongji University, China) for data collection.

\section{References}

[1] W. H. Wang, Vehicle's Man-Machine Interaction Safety and Driver Asistance, China Communications Press, Beijing, China, 2012.

[2] J. Du, J. Masters, and M. Barth, "Lane-level positioning for in-vehicle navigation and automated vehicle location (AVL) systems," in Proceedings of the 7th International IEEE Conference on Intelligent Transportation Systems (ITSC '04), pp. 35-40, Washington, DC, USA, October 2004.

[3] A. Boukerche, H. A. B. F. Oliveira, E. F. Nakamura, and A. A. F. Loureiro, "Vehicular Ad Hoc networks: a new challenge for localization-based systems," Computer Communications, vol. 31, no. 12, pp. 28382849, 2008.

[4] Q. He and K. Larry Head, Lane Level Vehicle Positioning With Low Cost GPS, Transportation Research Board, Washington, DC, USA, 2010.

[5] J. Du and M. J. Barth, "Next-generation automated vehicle location systems: positioning at the lane level," IEEE Transactions on Intelligent Transportation Systems, vol. 9, no. 1, pp. 48-57, 2008.

[6] D. Kubrak, C. Macabiau, M. Monnerat, and M. L. Bouchert, "Vehicular navigation using a tight integration of aided-GPS and low-cost MEMS sensors," in Proceedings of the Institute of Navigation, National Technical Meeting (NTM '06), pp. 149-158, January 2006.

[7] M. A. Quddus, W. Y. Ochieng, and R. B. Noland, "Current map-matching algorithms for transport applications: state-of-the art and future research directions," Transportation Research C, vol. 15, no. 5, pp. 312-328, 2007.

[8] R. Toledo-Moreo, D. Bétaille, and F. Peyret, "Lane-level integrity provision for navigation and map matching with GNSS, dead reckoning, and enhanced maps," IEEE Transactions on Intelligent Transportation Systems, vol. 11, no. 1, pp. 100-112, 2010.

[9] N. Uchitomi, A. Inada, M. Fujimoto, T. Wada, K. Mutsuura, and H. Okada, "Accurate indoor position estimation by Swift-Communication Range Recognition (S-CRR) method in passive RFID systems," in Proceedings of the International Conference on Indoor Positioning and Indoor Navigation (IPIN '10), Zürich, Switzerland, September 2010.

[10] H. Liu, H. Darabi, P. Banerjee, and J. Liu, "Survey of wireless indoor positioning techniques and systems," IEEE Transactions on Systems, Man and Cybernetics C, vol. 37, no. 6, pp. 1067-1080, 2007.

[11] E. K. . Lee, S. Y. Oh, and M. Gerla, "RFID assisted vehicle positioning in VANETs," Pervasive and Mobile Computing, vol. 8, no. 2, pp. 167-179.

[12] E. . Zhang, W. Jiang, Y. Kuang et al., "Active RFID positioning of vehicles in road Traffic," in Proceedings of the 11th International Symposium on Communications $\mathcal{E}$ Information Technologies (ISCIT '11), Hangzhou, China.

[13] X. G. . Yang, L. Y. Huang, Y. S. Wang, R. H. Du, J. W. Wang, and F. Yang, A prototype of a cooperative vehicle infrastructure system: proof of concept—case study in Tongji University, Transportation Research Board, ashington, DC, USA, 2012.

[14] S. E. Shladover and S. K. Tan, "Analysis of vehicle positioning accuracy requirements for communication-based cooperative collision warning," Journal of Intelligent Transportation Systems, vol. 10, no. 3, pp. 131-140, 2006.

[15] W. Y. Ochieng, M. A. Quddus, and R. B. Noland, "Map-matching in complex urban road networks," Brazilian Journal of Cartography, vol. 55, no. 2, pp. 1-18, 2004.

[16] L. . Sun, Y. Wu, J. Xu et al., "An RSU-assisted localization method in non-GPS highway traffic with dead reckoning and V2R communications," in Proceedings of the 2nd International Conference on Consumer Electronics, Communications and Networks (CECNet '12), Yichang, China, 2012. 


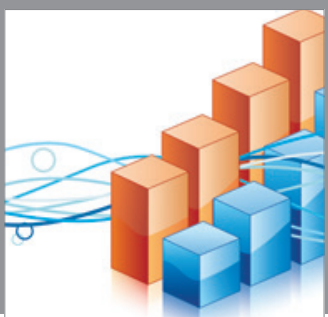

Advances in

Operations Research

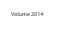

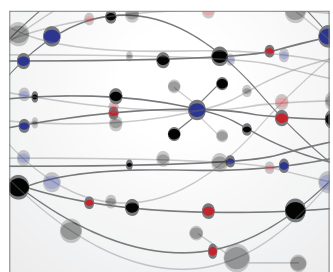

\section{The Scientific} World Journal
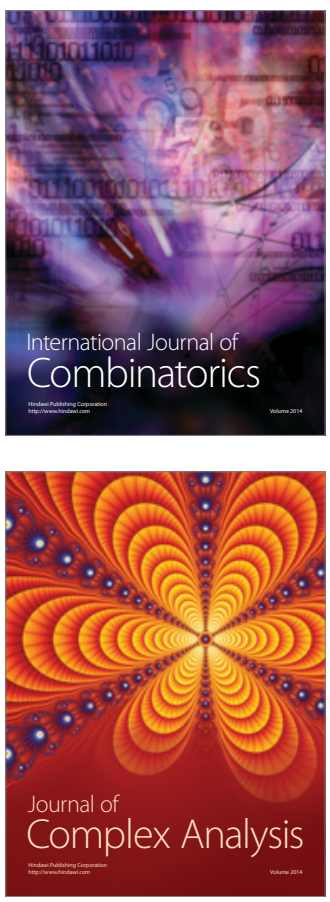

International Journal of

Mathematics and

Mathematical

Sciences
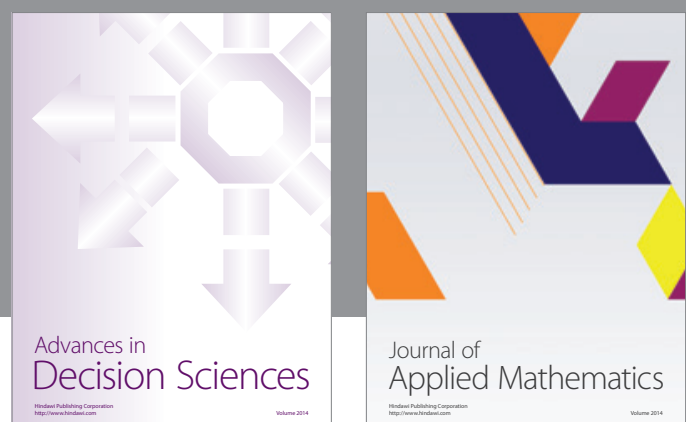

Journal of

Applied Mathematics
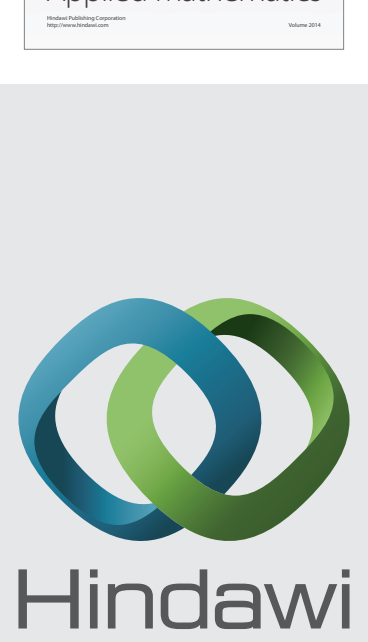

Submit your manuscripts at http://www.hindawi.com
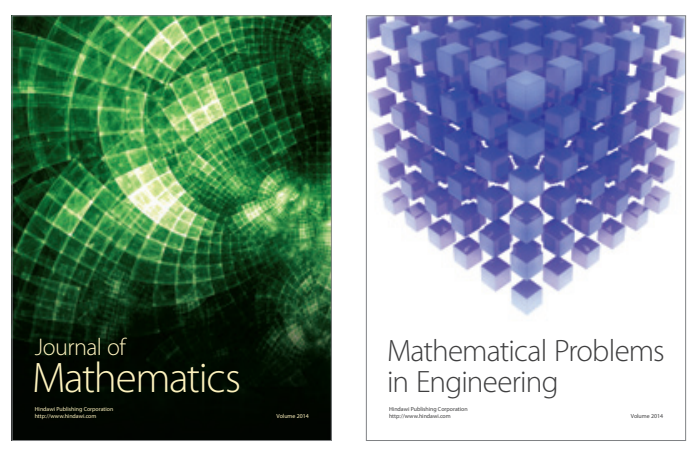

Mathematical Problems in Engineering
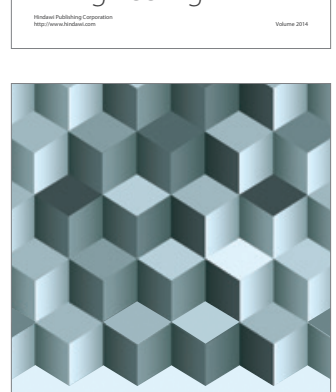

Journal of

Function Spaces
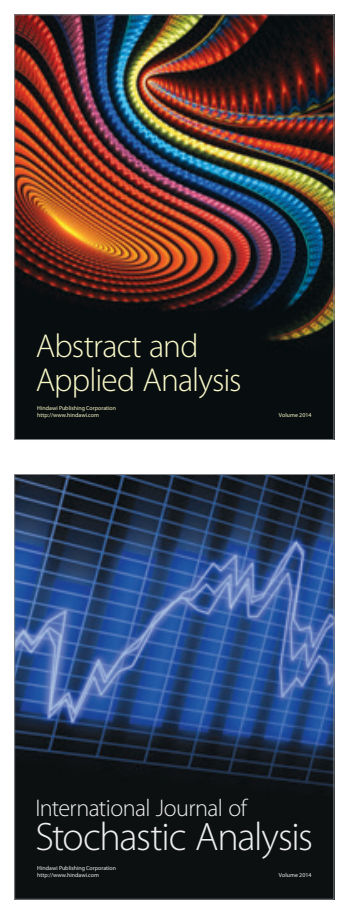

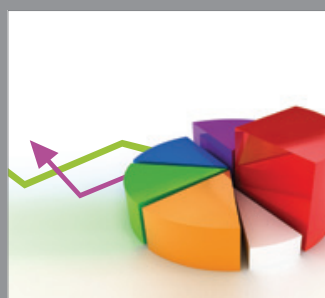

ournal of

Probability and Statistics

Promensencen
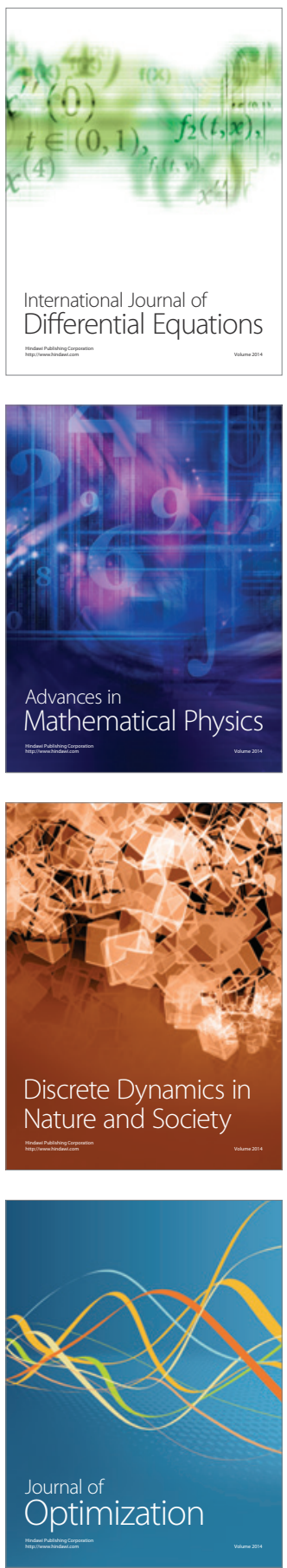